\title{
Characterisation of CASPR2 deficiency disorder - a syndrome involving autism, epilepsy and language impairment
}

\author{
Pedro Rodenas-Cuadrado', Nicola Pietrafusa ${ }^{2}$, Teresa Francavilla², Angela La Neve2 ${ }^{2}$ Pasquale Striano ${ }^{3}$ \\ and Sonja C. Vernes ${ }^{1,4^{*}}$ (D)
}

\begin{abstract}
Background: Heterozygous mutations in CNTNAP2 have been identified in patients with a range of complex phenotypes including intellectual disability, autism and schizophrenia. However heterozygous CNTNAP2 mutations are also found in the normal population. Conversely, homozygous mutations are rare in patient populations and have not been found in any unaffected individuals.

Case presentation: We describe a consanguineous family carrying a deletion in CNTNAP2 predicted to abolish function of its protein product, CASPR2. Homozygous family members display epilepsy, facial dysmorphisms, severe intellectual disability and impaired language. We compared these patients with previously reported individuals carrying homozygous mutations in CNTNAP2 and identified a highly recognisable phenotype.

Conclusions: We propose that CASPR2 loss produces a syndrome involving early-onset refractory epilepsy, intellectual disability, language impairment and autistic features that can be recognized as CASPR2 deficiency disorder. Further screening for homozygous patients meeting these criteria, together with detailed phenotypic and molecular investigations will be crucial for understanding the contribution of CNTNAP2 to normal and disrupted development.
\end{abstract}

Keywords: CNTNAP2, Epilepsy, Intellectual disability, Language regression, Autism

\section{Background}

The CNTNAP2 gene (located on 7q35) is encoded by 24 exons, spanning $2.3 \mathrm{Mb}$, making it a large physical target for disruptive mutations. The majority of reported patients carry heterozygous disruptions of CNTNAP2 and usually display some combination of core phenotypes including intellectual disability (ID), seizures, autistic features and impaired language [1]. However, the phenotype associated with heterozygous CNTNAP2 mutation is complex as the severity of characteristics varies across patients and in some cases additional phenotypes are observed (e.g. Tourette syndrome, schizophrenia). Furthermore, coding changes in a single copy of CNTNAP2 have also

\footnotetext{
*Correspondence: Sonja.Vernes@mpi.nl

${ }^{1}$ Max Planck Institute for Psycholinguistics, PO Box 310, Nijmegen 6500 AH, The Netherlands

${ }^{4}$ Donders Centre for Cognitive Neuroimaging, Kapittelweg 29, Nijmegen 6525 EN, The Netherlands

Full list of author information is available at the end of the article
}

been found in unaffected individuals, suggesting heterozygous mutations are not always fully penetrant.

CNTNAP2 encodes the CASPR2 protein - a member of the neurexin superfamily of proteins that facilitate cell-cell interactions [2] and form molecular bridges at synapses to influence synaptic strength and plasticity [3]. CASPR2 is a transmembrane protein with a small intracellular and a large extracellular component (see Fig. 1) containing four laminin G domains and two epidermal growth factor-like (EGF-like) domains thought to be involved in receptor-ligand interactions, cell adhesion, migration and differentiation $[4,5]$. Unlike most neurexin proteins, the CASPR2 extracellular region also contains a discoidin/neuropilin homology domain and a fibrinogen-like region which mediate cell-cell adhesions and extracellular matrix interactions [6].

CNTNAP2 is expressed across a number of brain regions throughout development and into adulthood (see [1] for review). In humans, CNTNAP2 is expressed in 
A

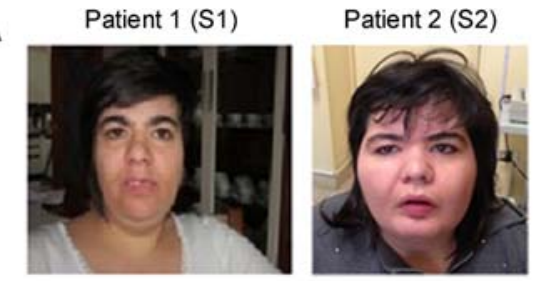

B

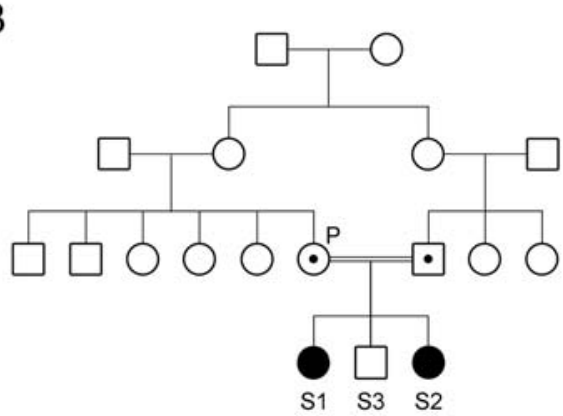

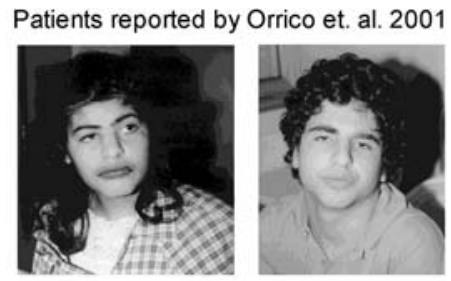

C
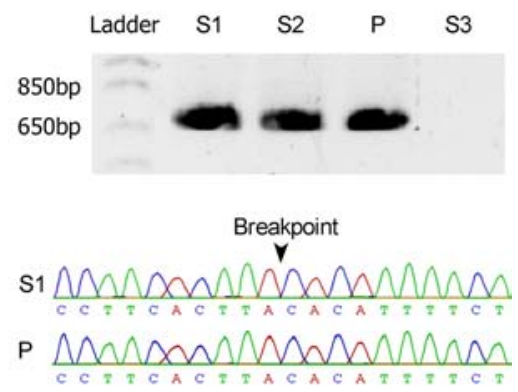

D

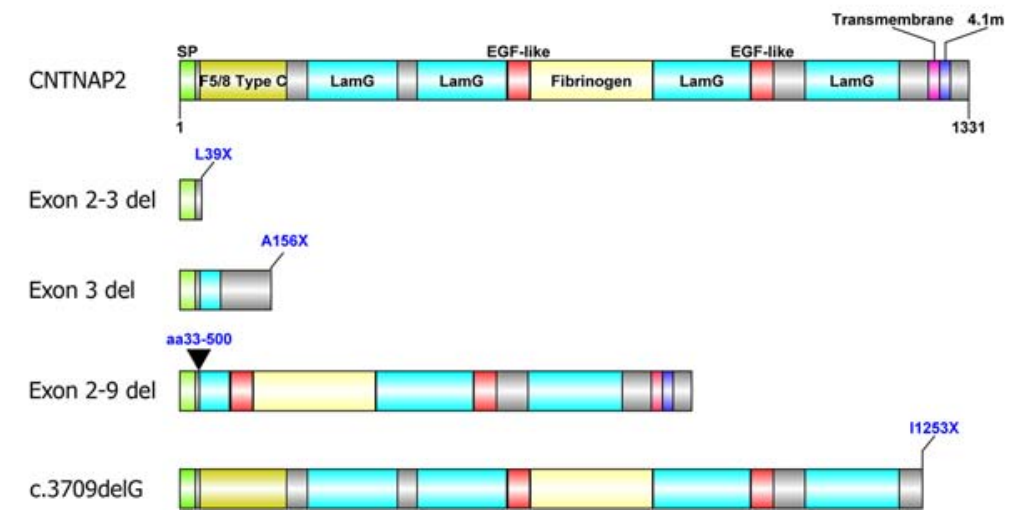

Fig. 1 a Clinical picture of the affected probands reported in this study. Note dysmorphic facial phenotype, low forehead and bushy eyebrows. Photos of the patients reported in Orrico et. al. are included for comparison (adapted from [18]) (b) Pedigree of the family showing the two affected siblings (filled symbols; S1 \& S2) with homozygous deletions encompassing exons 2-3 of the CNTNAP2 gene. The brother is unaffected (unfilled symbol; S3) and has no detected CNV's. Parents are first cousins and both carry the heterozygous CNTNAP2 deletion (symbols with dots; P), but are phenotypically normal. c PCR gel of the mutant CNTNAP2 gene. A 700 base pair product spanning the breakpoint could be amplified from the homozygous siblings and heterozygous parent, but not from the unaffected sibling. A Sanger sequencing trace shows the location of the deletion breakpoint. $\mathbf{d}$ The wild type CASPR2 protein and predicted functional consequences of CNTNAP2 patient mutations on the CASPR2 protein product. CASPR2 is composed of 1331 amino acids with a number of functional domains including a signal peptide (SP), discoidin/neuropilin homology domain (F5/8 Type C), Laminin G domain (LamG), Epidermal Growth Factor like domain (EGF-like), Fibrinogen domain (Fibrinogen), Transmembrane domain (Transmembrane), and Protein 4.1 homologous binding domain (4.1 m). The exon 2-3 deletion (reported herein), exon 3 deletion [16] and c3709delG mutation [14] all produce truncated proteins due to early stop codons. The exon 2-9 deletion [13] also produces a truncated protein product, however in this case it is due to an in-frame loss of amino acids 33-500

the cortex with enrichment in frontal and perisylvian regions, and in the striatum, thalamus and amygdala [7, 8]. This expression pattern recapitulates the cortico-striatothalamic circuitry known to modulate higher order cognitive processes, including language, reward, and frontal executive function. In mouse models, CASPR2 affects synaptic transmission and dendritic arborisation [9], and both mouse models and human patients have suggested that CNTNAP2 is important for normal cortical development [10]. Thus it is clear that CASPR2 plays an important role in brain development and function.

\section{Materials and methods \\ Patients}

Ethical approval for this study was obtained by the Ethics Committee of the University of Bari, Italy.

\section{Genetic study}

Array-CGH (aCGH) was performed using the BlueGnome ISCA $4 \times 44 k$ Cytochip oligoarray. Copy number variants (CNVs) were identified using the BlueFuse multi v2.3 software (BlueGnome). Human genomic DNA was used as a positive control (Promega \#G1471) [11]. A CNV was 
identified on chromosome 7. To confirm the deletion, DNA was extracted from blood samples of the affected sibs and family controls. PCR primers flanking the deletion were used to amplify DNA and determine the approximate deletion breakpoint. PCR was performed using Taq DNA polymerase kit (Invitrogen) as per supplier's instructions. Following this, a fragment spanning the breakpoint was amplified using the Advantage2 PCR kit (Clonetech, as per supplier's instructions), using the following primers; Fwd: TCCGGTCTTGGCATTGGGAA TCATTAGCC, Rev: TCAGCAGAGTGAAGAGACAAC CCACAGAGT. This breakpoint spanning PCR yielded a $730 \mathrm{bp}$ product from homozygous and heterozygous DNA but not from control samples. This product was then Sanger sequenced to identify the precise breakpoint.

\section{Case Presentation}

\section{Clinical report}

We report a consanguineous family carrying a deletion disrupting the coding region of CNTNAP2. Two affected female siblings (Patient 1 and 2) identified in this family were homozygous for the deletion (Fig. 1). The affected sisters had one unaffected male sibling that did not carry the deletion and their parents (first degree cousins) were both heterozygous for the deletion but were also unaffected. Patient 1 and 2 were born at full term displaying normal height, weight and head circumference with no reported perinatal problems. Since 2 years of age the weight of both patients has been consistently $>97^{\text {th }}$ percentile, with Patient 2 classified as severely obese. This has resulted in patient 2 having limited movement (awkward gait), however, neither sibling displays focal motor deficits of the limbs. Additionally, both siblings display a dysmorphic facial phenotype with low forehead and hairline, bushy eyebrows, high arched palate and dental malocclusion (Fig. 1a).

Psychomotor development was normal up to 36 months for patient 1 , and 18 months for patient 2 . At these points, the patients developed trunk swinging motor stereotypies and complex partial seizures. These seizures were characterised by loss of contact, expressions of fright and redness of the face with perioral cyanosis. Over time the seizures became more frequent and additional features developed including stertorous breathing, loss of consciousness and sometimes generalised tonic-clonic movement or falling to the ground. The seizures were rapidly and completely controlled by carbamazepine $(1000 \mathrm{mg} /$ day $)$ in patient 1 . However, for patient 2 carbamazepine treatment provided only a temporary decrease in the frequency of seizures and she ultimately became unresponsive to it as well as several other anticonvulsants (valproate, phenobarbital, vigabatrine, clobazam, lamotrigine and topiramate). Currently patient 2 has daily seizures of variable frequency (up to 4-5 episodes per day).
An EEG performed in patient 1 displayed poorly organised background activity without slow and epileptiform abnormalities. In contrast, patient 2 displayed short theta widespread outbursts with pseudo-periodic trend at 20 months of age. During sleep these outbursts were prevalent in the left hemisphere of the medial temporal region. At 4 years and 2 months of age, patient 2 performed another EEG. In this case, background activity was well organized, with outbursts of slow waves $(4 / 5 \mathrm{~Hz})$ sometimes preceded by small amplitude spikes in the right temporal region and spreading to temporal regions of the contralateral hemisphere. During sleep, the anomalies assumed the character of slow waves and slow spike-waves that spread from the right hemisphere to the contralateral side. Recorded seizures showed theta-delta waves and spikes in right temporal hemisphere and vertex, followed by $3 \mathrm{~Hz}$ slow activity spread throughout the right hemisphere. At 14 years old patient 2 underwent EEG exams during sleep and showed diffuse epileptiform abnormalities (spikes) in anterior bilateral regions.

An MRI scan of patient 1 did not identify any gross structural abnormalities. However, patient 2 displayed hypoplasia of the cerebellar vermis and hemispheres. Both patients have now been diagnosed with severe intellectual disability according to the Wechsler adult intelligence scale, and have also been classified with autistic spectrum disorder (DSM 5).

Language regression occurred simultaneously with seizure onset in both patients. For patient 1 , only syllables were initially lost followed by disappearance of verbal production. Currently, the probands language skills are limited to a few isolated words, rarely spoken with communicative intent. Patient 1 also displays vocal tics, reduced relational capacity and autonomy, together with increased stereotypies and clumsiness. Patient 2 had progressive behavioural changes with a slowing in language acquisition, impoverished interpersonal skills and verbal communication, including inertia and apathy. The patient's mother reported alternating periods of more pronounced symptoms, with periods in which seizures were better controlled and the child appeared to be more attentive and involved, but also more aggressive.

\section{Genetic analysis}

Molecular analysis of the patients was normal for karyotype (46XX) and negative for the presence of sub-telomeric deletions. Array-CGH revealed a deletion of at least $127 \mathrm{~Kb}$ in chromosome 7q35 (hg18; Chr7:146,082,310-146,189,705). Both affected probands were homozygous for the deletion, while the parents were heterozygous. The unaffected male sibling did not carry this deletion. The exact breakpoints were mapped via PCR and direct Sanger sequencing revealing a $203 \mathrm{~kb}$ deletion (hg38; Chr7:146,711,006-146,914,175) spanning exon 2-3 of the CNTNAP2 gene (Fig. 1c). 
CNTNAP2 expression was assayed in peripheral blood and in transformed lymphoblast cells, but expression levels were too low to reliably detect a signal in patient or control samples (data not shown (see also [12, 13]). Sequence analysis suggested that the loss of exons 2-3 introduces a frameshift into the CNTNAP2 transcript. This frameshift is predicted to produce an early stop codon at amino acid 39 (L39X) that would severely truncate the protein product and is likely to lead to nonsense mediated decay (Fig. 1d). This suggests that the CNV reported in these patients results in complete loss of functional CASPR2 protein in the homozygous state.

\section{Conclusions}

Herein we report two sisters with a homozygous deletion affecting the CNTNAP2 gene and a phenotype including dysmorphic features, severe intellectual disability, early onset seizures, autism spectrum disorder and language impairment. Heterozygous family members carrying this deletion were phenotypically normal. A search of publicly available exome sequencing data (ExAc database (http:// exac.broadinstitute.org/, Last accessed Jan 2016) did not find any homozygous coding changes in CNTNAP2 in the general population. One non-coding SNP in intron 12 (rs113545270) was reported to be homozygous in 76 of 5,077 exomes in the ExAc database. This variant was also reported as a possible loss-of-function change for an alternative transcript variant of CNTNAP2, however there is little evidence that this highly truncated variant is expressed. Furthermore this alternative transcript has been flagged as 'suspect' by the ensembl database (http://www.ensembl.org/ index.html), suggesting that this change does not affect the coding sequence of CNTNAP2.

Previously, only three other examples of homozygous loss-of-function CNTNAP2 mutations have been reported. The first of the reported homozygous CNTNAP2 deletions consisted of an old-order Amish cohort with a frameshift mutation (c.3709delG) in 18 individuals resulting in the introduction of an early stop codon towards the C-terminus of the CASPR2 protein (I1253X) (Fig. 1d) [14]. The protein produced from this mutation retains all the extracellular CASPR2 domains, but is no longer tethered to the neuronal cell membrane and is secreted, suggesting a loss of functional CASPR2 [15]. An unrelated consanguineous family was identified in which three members had a homozygous deletion of exon 3 within the CNTNAP2 locus [16]. The loss of exon 3 is predicted to introduce an early stop codon (A156X) due to a frameshift mutation. This is likely to result in nonsense mediated decay (NMD) of the RNA product and complete loss of CASPR2 protein in these individuals (Fig. 1d). Finally an in-frame homozygous deletion of CNTNAP2 exons 2-9 (del.33-500; Exon 2-9 del) was reported in two siblings of another unrelated family [17].
Deletion of these amino acids is predicted to result in the partial loss of extracellular domains (Fig. 1d). Molecular studies would be needed to determine if this mutant form of CASPR2 retains any functional characteristics, however the loss of almost half the extracellular domain of this protein is suggestive of a severe disruption of function. As in the family reported herein, heterozygous family members were identified in each of these three groups that were phenotypically normal.

In all cases where CASPR2 deficiency was identified, individuals displayed severe intellectual disability and early onset of severe seizures, beginning by 13-36 months of age and often associated with speech/language and behavioural regression (Table 1). Most patients displayed seizures that were refractive to pharmacological or surgical treatments. Treatment was successful only in 3 patients. In all patients, seizures were severe and frequent, with multiple episodes per day being reported in all cases. The early seizure onset observed in these patients presents difficulties in determining if the speech and language impairments observed are directly due to CNTNAP2 mutation or secondary to seizures. For example in I1253X patients, language comprehension was reported to be good before seizure onset around 13-20 months, but all patients showed language regression from 36 months. However, in contrast, in the A156X family the male child did not speak or vocalise, even before seizure onset. Similarly, the del.33-500 siblings only developed a few isolated words. The patients we report herein (L39X) showed language regression occurring simultaneously with seizure onset, until speech became limited to the rare use of isolated words. Taken together, these families suggest that CNTNAP2 loss results in seizures that may cause language regression, but that it may also be affecting the development of regions of the brain involved in speech and language as impairments in these faculties can occur before seizure onset.

All patients have also been reported to have some autistic traits. Both our patients are classified as autistic with poor communication skills, impaired communication, repetitive behaviour, and motor stereotypies. The majority of patients reported with the I1253X mutation were classified as autistic, displaying behavioural deterioration including restricted behaviours, aberrant social interactions and communicative impairment [14]. Zweier et al. noted that the del.33-500 siblings had limited communicative ability with variable "autistic behaviour and stereotypic movements" [13] and Watson et al. reported that A156X siblings displayed obsessive compulsive behaviour, stereotypical movements and communicative and speech impairments [16]. Given that CNTNAP2 remains a top candidate gene with regards to the molecular basis of autism, these homozygous patients would be of great value for studying the links between CNTNAP2 and autism. Thus there is a need for further in-depth phenotyping of 
Table 1 Physical, neurodevelopmental and behavioural features of patients identified with homozygous CNTNAP2 mutations resulting in CASPR2 deficiency disorder

\begin{tabular}{|c|c|c|c|c|c|}
\hline & & $\begin{array}{l}\text { Strauss et al., 2006, } \\
\text { Jackman et al., } 2009\end{array}$ & $\begin{array}{l}\text { Zweier et al., } 2009 \\
\text { Orrico et al., } 2001\end{array}$ & Watson et al., 2014 & This study \\
\hline Patients & & 18 & 2 & 3 & 2 \\
\hline \multirow[t]{3}{*}{ Mutation } & DNA variant & c.3709delG & CNV del ex 2-9 & CNV del ex 3 & CNV del ex 2-3 \\
\hline & Effect & Early STOP codon & In frame deletion & Frameshift, early STOP codon & $\begin{array}{l}\text { Frameshift, } \\
\text { early STOP codon }\end{array}$ \\
\hline & Resulting protein & I1253X & del.33-500 & A156X & L39X \\
\hline \multirow[t]{5}{*}{ Development } & Pregnancy & NR & Normal & Normal & Normal \\
\hline & Intellectual disability & Severe & Severe & Severe & Severe \\
\hline & Psychomotor delay & + & Mild & + & + \\
\hline & Gross motor delay & + & Mild & + & - \\
\hline & Ataxic cerebral palsy & NR & $N R$ & + & - \\
\hline \multirow[t]{6}{*}{ Morphology } & Dysmorphic & NR & + & Mild & + \\
\hline & Low forehead/hairline & NR & + & $N R$ & + \\
\hline & Arched palate & NR & - & $N R$ & + \\
\hline & Dental malocclusion & NR & - & $N R$ & + \\
\hline & Obesity & NR & - & NR & + \\
\hline & Gait & NR & NR & Broad-based ataxic gait & $\begin{array}{l}\text { Awkward gait } \\
\text { (only one sib) }\end{array}$ \\
\hline \multirow[t]{8}{*}{ Behaviour } & Autism & + & - & - & + \\
\hline & Autistic features & + & + & + & + \\
\hline & Motor stereotypies & NR & + & + & + \\
\hline & Communicative impairment & + & + & + & + \\
\hline & Behavioural problems & + & + & + & + \\
\hline & Inertia/apathy & NR & NR & NR & + \\
\hline & Agression & + & NR & $N R$ & + \\
\hline & $\mathrm{ADHD}$ & + & NR & restlessness & - \\
\hline \multirow[t]{3}{*}{ Epilepsy } & Seizures & Yes & Yes & Yes & Yes \\
\hline & Age onset (months) & $13-20$ & $22-30$ & 24 & $20-36$ \\
\hline & Therapy & Refractive & Refractive & Carbamazepine or valproate & $\begin{array}{l}\text { P1 - Carbamazepine, } \\
\text { P2 - Refractive }\end{array}$ \\
\hline \multirow[t]{3}{*}{ Language } & Language impairment/regression & + & + & + & + \\
\hline & Vocal tics & NR & NR & NR & + \\
\hline & Limited/absent language & + & + & + & + \\
\hline Neurological & Dysplasia & + & + & NR & + \\
\hline \multirow[t]{4}{*}{ Breathing/other } & Hyperventilation & NR & + & NR & - \\
\hline & Apnea & NR & +/- (only one sib) & NR & - \\
\hline & Cyanoses & NR & +/- (only one sib) & NR & + \\
\hline & Loss of consciousness & NR & +/- (only one sib) & NR & + \\
\hline
\end{tabular}

NR Not reported

the autistic characteristics of the homozygous patients in these families.

Additional common features between the probands include dysmorphisms, focal cortical dysplasia, behavioural problems and psychomotor delay (Table 1). Dysmorphic features were reported from three of the four mutations (del.33-500, A156X and L39X) and there were striking similarities between the del.33-500 patients and our patients, i.e. coarse facial features, low forehead, heavy eyebrows and large mouth with thick lips (Fig. 1a) [18]. In 
future, it will be of interest to determine if these mild dysmorphia are a common characteristic of CASPR2 deficiency.

Our patients did not show any clinical symptoms suggestive of peripheral nerve hyperexcitability (e.g. myokymia, hyperhidrosis) or neuropathy (e.g. sensory/ motor dysfunction). However, given that CAPSR2 is required for the clustering of juxtaparanodal potassium channels [5] and that anti-CASPR2 antibodies have been associated with acquired peripheral nerve excitability [19], late onset of peripheral nerve involvement cannot be excluded a priori and nerve conduction velocity/ electromyography may be warranted in the future.

In summary, while some variability in phenotype remains, it is clear that homozygous loss of CNTNAP2 results in a syndromic disorder characterised by severe intellectual disability, early-onset drug-resistant epilepsy, reduced or absent language, communicative impairments and autistic phenotypes. To further refine the diagnostic criteria for CASPR2 deficiency disorder it will be essential to identify novel patients carrying homozygous CNTNAP2 mutations by screening cohorts showing core syndromic phenotypes, together with a more detailed phenotypic analysis of existing and novel patients. Furthermore, although the mutations discussed above are predicted to result in a loss of CASPR2 function, cell based and molecular assays will be critical to determine, which mutations represent a full vs. partial loss of function. This is particularly relevant for mutations such as del.33-500 or I1253X where truncated forms of the protein, retaining some functional domains, are predicted. However, these assays should seek patient samples beyond blood or lymphoblasts as we and others have shown that CNTNAP2 cannot be reliably detected in these cell types. Determining which mutations represent a true loss of function together with increased phenotypic analysis will be of great value in refining the phenotype associated with CASPR2 deficiency disorder, and in understanding the contribution of CNTNAP2 to cognitive development as well as disorders such as epilepsy, autism and language impairment.

\section{Consent}

Written informed consent was obtained from the parent of the patients for publication of this Case report and any accompanying images. A copy of the written consent is available for review by the Editor of this journal.

\section{Competing interests}

The authors declare that they have no competing interests.

\section{Authors' contributions}

PRC carried out the molecular genetic studies, NP collected patient samples, TF and AN phenotyped the patients and contributed to the manuscript, PS analysed data and drafted the manuscript and PRC and SCV designed the study, analysed data and drafted the manuscript. All authors read and approved the final manuscript.

\section{Acknowledgments}

This work was supported by a Marie Curie Career Integration Grant awarded to S.C.V. and by the Max Planck Society. We are grateful to the family for their consent in undertaking this study.

\section{Author details}

${ }^{1}$ Max Planck Institute for Psycholinguistics, PO Box 310, Nijmegen 6500 AH, The Netherlands. ${ }^{2}$ Department of Basic Medical Sciences, Neurosciences and Sense Organs, University of Bari, Bari, Italy. ${ }^{3}$ Pediatric Neurology and Muscular Diseases Unit, Department of Neurosciences, Rehabilitation, Ophthalmology, Genetics, Maternal and Child Health, University of Genoa, "G. Gaslini" Institute, Genoa, Italy. ${ }^{4}$ Donders Centre for Cognitive Neuroimaging, Kapittelweg 29, Nijmegen 6525 EN, The Netherlands.

Received: 23 July 2015 Accepted: 29 January 2016

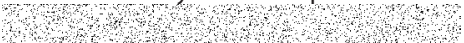

\section{References}

1. Rodenas-Cuadrado P, Ho J, Vernes SC. Shining a light on CNTNAP2: complex functions to complex disorders. Eur J Hum Genet. 2014:22(2):171-8.

2. Nakabayashi $\mathrm{K}$, Scherer SW. The human contactin-associated protein-like 2 gene (CNTNAP2) spans over $2 \mathrm{Mb}$ of DNA at chromosome 7q35. Genomics. 2001;73(1):108-12.

3. Fisher SE, Vernes SC. Genetics and the Language Sciences. Annu Rev Linguist. 2015;1(1):null.

4. Traka M, Goutebroze L, Denisenko N, Bessa M, Nifli A, Havaki S, et al. Association of TAG-1 with Caspr2 is essential for the molecular organization of juxtaparanodal regions of myelinated fibers. J Cell Biol. 2003;162(6):1161-72.

5. Poliak S, Salomon D, Elhanany H, Sabanay H, Kiernan B, Pevny L, et al. Juxtaparanodal clustering of Shaker-like K+ channels in myelinated axons depends on Caspr2 and TAG-1. J Cell Biol. 2003;162(6):1149-60.

6. Poliak S, Gollan L, Martinez R, Custer A, Einheber S, Salzer UL, et al. Caspr2, a new member of the neurexin superfamily, is localized at the juxtaparanodes of myelinated axons and associates with K+ channels. Neuron. 1999;24(4):1037-47.

7. Alarcon M, Abrahams BS, Stone JL, Duvall JA, Perederiy JV, Bomar JM, et al. Linkage, association, and gene-expression analyses identify CNTNAP2 as an autism-susceptibility gene. Am J Hum Genet. 2008;82(1):150-9.

8. Abrahams BS, Tentler D, Perederiy JV, Oldham MC, Coppola G, Geschwind DH. Genome-wide analyses of human perisylvian cerebral cortical patterning. Proc Natl Acad Sci U S A. 2007;104(45):17849-54.

9. Anderson GR, Galfin T, Xu W, Aoto J, Malenka RC, Sudhof TC. Candidate autism gene screen identifies critical role for cell-adhesion molecule CASPR2 in dendritic arborization and spine development. Proc Natl Acad Sci USA. 2012;109(44):18120-5.

10. Peñagarikano O, Abrahams Brett S, Herman Edward I, Winden Kellen D, Gdalyahu A, Dong H, et al. Absence of CNTNAP2 Leads to Epilepsy, Neuronal Migration Abnormalities, and Core Autism-Related Deficits. Cell. 2011;147(1):235-46.

11. Striano P, Coppola A, Paravidino R, Malacarne M, Gimelli S, Robbiano A, et al. Clinical significance of rare copy number variations in epilepsy: a casecontrol survey using microarray-based comparative genomic hybridization. Arch Neurol. 2012;69(3):322-30.

12. Bakkaloglu B, O'Roak BJ, Louvi A, Gupta AR, Abelson JF, Morgan TM, et al. Molecular cytogenetic analysis and resequencing of contactin associated proteinlike 2 in autism spectrum disorders. Am J Hum Genet. 2008;82(1):165-73.

13. Zweier $\mathrm{C}$, de Jong EK, Zweier M, Orrico A, Ousager LB, Collins AL, et al. CNTNAP2 and NRXN1 Are Mutated in Autosomal-Recessive Pitt-Hopkins-like Mental Retardation and Determine the Level of a Common Synaptic Protein in Drosophila. Am J Hum Genet. 2009:85(5):655-66.

14. Strauss KA, Puffenberger EG, Huentelman MJ, Gottlieb S, Dobrin SE, Parod $J \mathrm{M}$, et al. Recessive symptomatic focal epilepsy and mutant contactinassociated protein-like 2. N Engl J Med. 2006:354(13):1370-7.

15. Falivelli G, De Jaco A, Favaloro FL, Kim H, Wilson J, Dubi N, et al. Inherited genetic variants in autism-related CNTNAP2 show perturbed trafficking and ATF6 activation. Hum Mol Genet. 2012;21(21):4761-73.

16. Watson CM, Crinnion LA, Tzika A, Mills A, Coates A, Pendlebury M, et al. Diagnostic whole genome sequencing and split-read mapping for nucleotide resolution breakpoint identification in CNTNAP2 deficiency syndrome. Am J Med Genet A. 2014;164A(10):2649-55. 
17. Gregor A, Albrecht B, Bader I, Bijlsma EK, Ekici AB, Engels H, et al. Expanding the clinical spectrum associated with defects in CNTNAP2 and NRXN1. BMC Med Genet. 2011;12:106.

18. Orrico A, Galli L, Zappella M, Lam CW, Bonifacio S, Torricelli F, et al. Possible case of Pitt-Hopkins syndrome in sibs. Am J Med Genet. 2001;103(2):157-9.

19. Lancaster E, Huijbers MG, Bar V, Boronat A, Wong A, Martinez-Hernandez E, et al. Investigations of caspr2, an autoantigen of encephalitis and neuromyotonia. Ann Neurol. 2011;69(2):303-11.

Submit your next manuscript to BioMed Central and we will help you at every step:

- We accept pre-submission inquiries

- Our selector tool helps you to find the most relevant journal

- We provide round the clock customer support

- Convenient online submission

- Thorough peer review

- Inclusion in PubMed and all major indexing services

- Maximum visibility for your research

Submit your manuscript at www.biomedcentral.com/submit 